\title{
Essence of Inviscid Shear Instability: a Point View of Vortex Dynamics
}

\author{
L. $\operatorname{Sun}^{1,2 *}$
}

${ }^{1}$ School of Earth and Space Sciences, University of Science and Technology of China, Hefei 230026, China

${ }^{2}$ LASG, Institute of Atmospheric Physics, Chinese Academy of Sciences, Beijing 100029, China.

Email: sunl@ustc.edu.cn; sunl@ustc.edu

\begin{abstract}
The essence of shear instability is fully revealed both mathematically and physically. A general sufficient and necessary stable criterion is obtained analytically within linear context. It is the analogue of Kelvin-Arnol'd theorem, i.e., the stable flow minimizes the kinetic energy associated with vorticity. Then the mechanism of shear instability is explored by combining the mechanisms of both KelvinHelmholtz instability (K-H instability) and resonance of waves. It requires both concentrated vortex and resonant waves for the instability. The waves, which have same phase speed with the concentrated vortex, have interactions with the vortex to trigger the instability. The physical explanation of shear instability is also sketched. These results would intrigue future works to investigate the other hydrodynamic instabilities.
\end{abstract}

\section{REFERENCES}

1. Drazin PG, Reid WH. Hydrodynamic Stability. Cambridge: Cambridge University Press, U.K. 1981

2. Huerre P, Rossi M. Hydrodynamic Instabilities in Open Flow In: Godreche C, Manneville P, eds. Hydrodynamics and nonlinear instabilities, Cambridge, Cambridge University Press: 1998, pp. $81-242$

3. Criminale WO, Jackson TL, Joslin RD. Theory and Computation of Hydrodynamic Stability. Cambridge, Cambridge University Press: U.K., 2003

4. Arnold V, Khesin B. Topological Methods in Hydrodynamics. Springer, 1998

5. Saffman PG. Vortex Dynamics. Cambridge, Cambridge University Press: U.K., 1992.

6. Vladimirov VA, Ilin KI. On Arnold's variational principles in fluid mechanics. In: Bierstone E, eds. The Arnoldfest, American Mathematical Society, 1999, 471-495

7. Sun L. General stability criterion of inviscid parallel flow. Arxiv. 2006; physics/0601043

8. Ponta FL, Aref H. Strouhal-Reynolds number relationship for vortex streets. Phys. Rev. Lett. 2002;93:084501

9. Vallis GK. Atmospheric and Oceanic Fluid Dynamics. Cambridge, Cambridge University Press: U. K., 2006.

10. Batchelor GK. An Introduction to Fluid Dynamics. Cambridge, Cambridge University Press: U. K., 1967

11. Baines P, Mitsudera H. On the mechanism of shear flow instabilities. J. Fluid Mech., 1994:276:327342 